\title{
Validation of a High-Resolution 3D Face Scanner Based on Stereophotogrammetry
}

\author{
L.M. GALANTUCCI, F. LAVECCHIA, G. PERCOCO, S. RASPATELLI \\ Politecnico di Bari, Dipartimento di Ingegneria Meccanica e Gestionale \\ Laboratorio di Prototipazione Rapida e Reverse Engineering, Bari, Italy
}

\begin{abstract}
Emerging 3D surface acquisition technologies have recently introduced new face scanning methodologies. Among passive methods, stereophotogrammetry has proved to be particularly promising in the evaluation of facial morphology. The aim of the current study is to propose and study a new and structured validation method for 3D face scanning system based on close-range stereophotogrammetry. The authors investigated accuracy of the FaceshapeMaxi5 3D photogrammetric scanner developed by Polishape 3D srl (spin-off of Politecnico di Bari). Measurements were taken over a set of 23 anthropological soft-tissue facial landmarks marked on two different dummies. The validation procedure consisted in: 1) determining the most appropriate photo-shooting parameters; 2) assessing the photogrammetric software precision; 3 ) testing the system measurements against those of a CMM. Critical was the camera system calibration process. A new method was implemented, namely full-field calibration. Accuracy was evaluated using different indicators. The operator error was measured by repeatedly digitising landmarks on the 3D model and it was within $0.059 \mathrm{~mm}$. The reproducibility error was calculated by digitising landmarks on two different occasions. The Euclidean distance between the two matched sets of coordinates was thus computed resulting within $0.090 \mathrm{~mm}$. Each dummy was digitised using a CMM of documented accuracy $(0.5 \mu \mathrm{m})$. The so obtained landmark coordinates were considered as the "gold standard". After Procrustes alignment, the displacement between the adjusted system coordinates and the "gold standard" was calculated. Therefore, the achieved results proved that the presented 3D face scanner was reliable enough for capturing facial morphology for clinical treatment and anthropological practice. The validation method is applicable to every kind of 3D Facial Scanner, requiring uniquely the presence of the textured and tessellated point cloud.
\end{abstract}

Keywords: Passive methods, photogrammetry, validation.

\section{Introduction}

Optical methods are probably the most popular systems because they are characterized a by higher acquisition speed than those of contact methods. Besides, optical scanners are particularly suitable for the acquisition of easily deformable surfaces, such as the human skin, and are relatively cheap compared to 3D active scanners. 3D image acquisition systems are becoming increasingly more affordable thanks to the large-scale deployment of digital cameras and to the great advances in computer graphics, each and every technology bearing its own pros and cons.

3D laser scanners are potentially very accurate and have been widely used in recent years. A 2005 study conducted by Kau et al.[1] intended to evaluate the reliability of this technique for the measurement of facial morphology. More specifically, they found out that the reproduction of facial morphology is accurate to within $0.85 \mathrm{~mm}$.

However, a 3D laser scanning system consists of delicate light sensors, high-quality optics and precise electric motors. Therefore, the high costs for producing hardware components have to be carefully taken into account. Moreover, the complete scanning system has to be calibrated so that the geometrical setup of all elements can be exactly determined. Another major drawback of this technology when applied to face scanning, lies in the acquisition time. As a matter of fact, this facet would not represent any issue if feet or hands had to be scanned. It is indeed possible for an individual to keep these body parts steady for at least a few seconds. As for such a non-static object like the human face, uncontrolled movements are inevitable. Even breathing or small unintentional muscle contractions might therefore be cause of error.

Photogrammetry currently provides the most cost-effective 3D scanning techniques. Indeed, the equipment needed for a photogrammetric scanner is quite simple: one or more digital cameras, an optional projector device and a photogrammetric software. Different setups have been explored depending on the number and types of cameras, using or not special coded targets and varying the light conditions. 
Technical literature provides numerous examples of comparative studies mainly between laser and photogrammetric scanners. Ferrandes et al. compared the scans of a Kreon KLS51 laser scanner and those obtained with a single-camera photogrammetric scanner for facial recognition [2].

In 2007 Setan et al. [3] gave further evidence that laser scanning provides rapid 3D modelling but photogrammetry precise measurement.

A similar study was conducted in [4-5]. The employed equipment consisted of an on-line Konica Minolta Vivid910i laser scanner, three 10-megapixel-CMOS sensors of commercial cameras and the powerful photogrammetric software PhotoModeler ${ }^{\circledR}$ 5.0. The maximum linear difference between measured distances was equal to $1.4 \mathrm{~mm}$, while the mean value was equal to $0.6 \mathrm{~mm}$.

Even structured-light technology has been compared to other digitisation techniques in order to validate newly developed 3D structured-light scanners in reconstructing facial morphology.

A recent study has been conducted by Ma et al. in 2009 [6]. The validation was performed in three facets including accuracy, precision and reliability. Accuracy and precision were investigated using a plaster model with 19 marked landmarks. Accuracy was determined by comparing the 3D point coordinates evaluated from the 3D reconstructions and those determined by a coordinate-measuring machine (CMM). Precision was quantified through the repeated landmarks location on 3D images. The results they obtained assured an accuracy of $0.93 \mathrm{~mm}$ and a precision of $0.79 \mathrm{~mm}$.

Another similar study conducted by Enciso et al. [7] focused on the development of indirect 3D landmark location and measurement of facial soft-tissue with the structured-light technique. The lightbased imaging system from Eyetronics consisted of a pattern projector and a digital camera. Statistical evaluation and validation of a 3D image-based face modelling technique was pursued using a mannequin head with pre-labelled anthropometric markers. 23 red-coloured markers stuck on the plaster face were used. These measurements were tested against a validated 3D digitizer, namely MicroScribe 3DX (Immersion Corp.). Since the digitizer was assumed as the "gold standard", differences between homologous measurements defined the imaging error. The overall mean absolute error of the structured-light scanner combining frontal, left and right side of the dummy amounted to $0.48 \mathrm{~mm}$, with maximum absolute error of $1.55 \mathrm{~mm}$.

Derya Germec-Cakan et al [8] made a comparison of facial soft tissue measurements done on plaster casts using laser scanners and photogrammetry, stating that laser scanning is not sensitive enough to visualise deeper indentations, and that stereophotogrammetry gives better results.

The accuracy of photogrammetric systems has been investigated by many authors, using special specimen and mannequins, and considering different types or errors (due to operator, capture position, capture system, calibration, registration).

Lee et al [9] investigated on a Rainbow 3D camera system using 6 landmarks on a pre-calibrated and a mannequin model, acquired from different angulations, recording the images at different times, having errors in length less than $1 \mathrm{~mm}$.

Ayoub et al [10] compared the result of measures obtained on plaster casts done both with CMM machines and $\mathrm{C} 3 \mathrm{D}^{\mathrm{TM}} 3 \mathrm{D}$ imaging system, considering different types of errors (operator, capture, registration) on 5 points, reporting a total error on average less than $1 \mathrm{~mm}$. In [11] they assessed the feasibility of building a virtual human face digitally by superimposing a photorealistic 3D soft-tissue surface on bone in the correct relationship and evaluating the registration errors. The 3D soft-tissue surface of the face was captured using a Di3D ${ }^{\mathrm{TM}}$ stereophotogrammetry system and the underlying bone was recorded using a 3D CT scanner. Using the Procrustes registration method, the outer surface of the 3D CT scan and the photorealistic soft-tissue surfaces were merged into a single Virtual Reality Modelling Language (VRML) file. Quantitative measurements of registration errors were calculated in the reconstructed human head models using the signed closest point distance from the photo-realistic skin surface to the transformed CT skin surface. The registration errors between most parts of the aligned surfaces were within $\pm 1.5 \mathrm{~mm}$, relatively larger around the eyebrows, eyelids and cheeks.

Boyadjiev [12] followed a similar approach though focusing on the morphometric analysis of nonsyndromic craniosynostosis. The novel approach consisted in collecting and analysing clinical and epidemiologic data from a library of soft-tissue surface scans obtained through photogrammetric $3 \mathrm{dMD}^{\mathrm{TM}}$ technology, as well as digital files of patients' head CT scans for $3 \mathrm{D}$ reconstruction and morphometric analysis. In the same field of congenital craniofacial malformations, Hammond et al. [13] evaluated the use of $3 D$ stereophotogrammetry to the analysis of face shape in Noonan Syndrome for different age bands.

Rangel et al. [14] presented a feasibility study concerning the integration of a digital dental cast into a 3D facial model. Three digital data sets were constructed: a digital dental cast, a digital 3D model of the patient with the teeth visible using $3 \mathrm{dMD}^{\mathrm{TM}}$ stereophotogrammetric system, and a digital 3D model of the patient with the teeth in occlusion. By using a special iterated closest point algorithm, these 
three data sets were matched to place them in the correct anatomical position. After matching, they obtained a 3D digital model with the dental cast visible through the transparent picture of the patient's face. When the distance between the matched data sets was calculated, an average distance of $0.35 \mathrm{~mm}$ with standard deviation of $0.32 \mathrm{~mm}$ was shown. These results can be regarded as clinically acceptable.

Khambay et al [15] validated an high resolution 3D imaging system (Di3 $\mathrm{D}^{\mathrm{TM}}$ ) using 10 landmarks on facial plaster casts, comparing the results with those obtained by a CMM, reporting a system error of $0.2 \mathrm{~mm}$ and a reproducibility error less than $0.5 \mathrm{~mm}$.

Winder et al [16] used 18 landmarks on a mannequin head to validate a Di3D ${ }^{\mathrm{TM}}$ system, using as comparison laser scans and digital calliper measures, having mean differences between measures of $0.62 \mathrm{~mm}$.

In 2010 Lübbers et al [17] examined the precision and accuracy of the $3 \mathrm{dMD}^{\mathrm{TM}}$ system using 41 landmarks on a mannequin head: they report a mean global error of $0.2 \mathrm{~mm}$ (range $0.1-0.5 \mathrm{~mm}$ ). More recently [18] they repeated the experiments using 61 landmarks on two real faces, reporting a mean global error of $0.41 \mathrm{~mm}$ (range $0 .-3.3 \mathrm{~mm}$ ).

The authors compared the results achieved with various photogrammetric systems developed by themselves: with up to three digital cameras of different brands and characteristics [19-20], with four and five DSRLs. They have recently also developed a simple photogrammetric system for automatic capture and measurement of facial soft tissues during movement [21]. Digital close-range photogrammetry was used to acquire the spatial coordinates of facial landmark points and track their movements.

\section{System}

The stereophotogrammetric system is composed of five DSLR cameras, namely four Canon EOS 40D and one Canon EOS 400D with a resolution of 10.1 Mpixels, mounted on a tripod support (Fig. 1). Three external flashes in addition to one internal flash enable the best uniform illumination conditions on the face surface. Fast and simultaneous photo-shooting are essential to achieve accurate results irrespective of possible subtle movements of the subject's head. The only condition subjects would stand is Natural Head Position (NHP). In this study, photographs were yet taken at a shooting time value of $1 / 100$ s. Five simultaneous photographs are taken for each subject, the central one for orientation purposes only. Synchronicity is controlled both on hardware and software level. The core processing software is PhotoModeler® Scanner by EOS Systems Inc. updated to version 6.3. 3D point reconstruction is aided by a coded-target poster placed behind the subject. The pre-requisite for precise measurements was the system calibration. A 3D full-field calibration was implemented so as to solve the resectioning problem. The calibrator consisted of a $3 \mathrm{D}$ object measuring $250 \times 170 \mathrm{~mm}$ with 4 levels on the Z-axis. Thus, a file called calibration certificate encapsulated all information about the P camera matrix and optical distortion. It was possible to evaluate and consequently adjust the calibration results by using two quality indicators PhotoModeler® Scanner is equipped of. The achieved values are: 0.0319 pixel as maximum residual, and 0.0160 pixel as root mean square (RMS) residual.

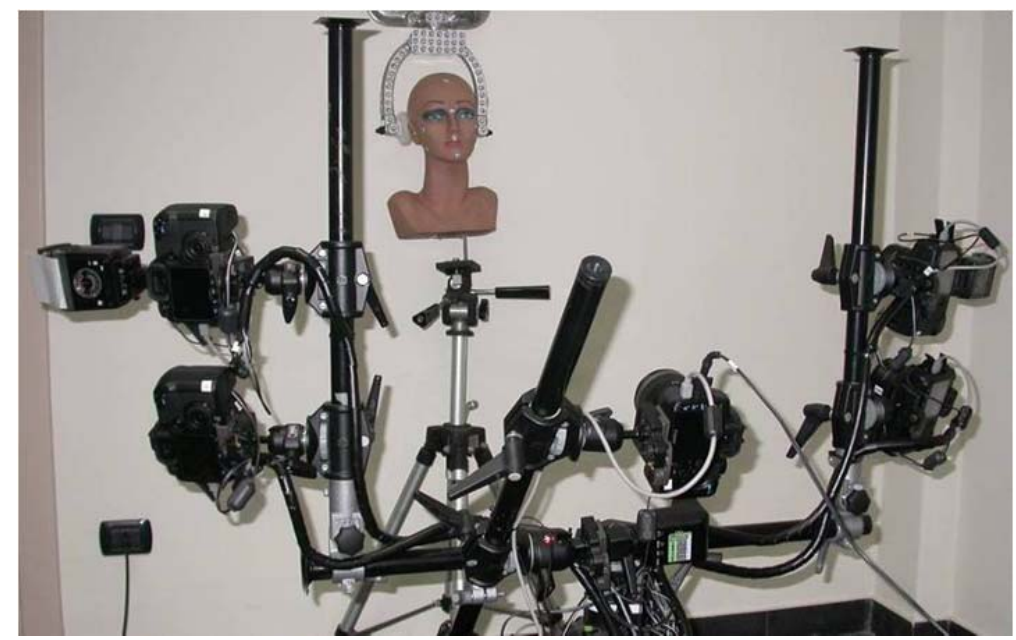

Fig. 1. The FaceshapeMaxi5 3D photogrammetric scanner system developed by Polishape 3D srl 


\section{Materials}

The present study focuses on developing an indirect 3D landmark location and measurement technique of facial soft tissues with close-range photogrammetry. Therefore, statistical evaluation and validation of the 3D scanning system was conducted using "fake" faces. For this purpose, two different mannequin heads were used. Being artificial artefacts, they were conceived so as to provide more exhaustive information about face morphology. For aesthetic reasons they were manufactured with a high surface finish. The female dummy, hereafter referred to as 'Mary', is a plaster model mounted on a plasterboard base. The male dummy, hereafter referred to as 'Jack', is made of white expanded polystyrene. They were both preliminary coloured with acrylic paint brushstrokes in order to resemble true skin complexion. Non-uniform manual coat of paint solved the random skin-like texture as well as the light reflectance problems.

\section{Method}

The objective pursued in this study was to validate the newly conceived 3D stereophotogrammetric face scanner through the analytical comparison of its measurements with those obtained from a $\mathrm{CMM}$. The general method followed by the authors is simplified in Fig. 2. The input data were the 3D coordinates of facial landmarks, both from the CMM and from the photogrammetric scanner. Data were collected repeatedly at different times in order to provide useful information about the reproducibility performance. The CMM produced 3 measurements for each landmark for each dummy in two separate weeks thus resulting in a total of 276 digitisations. By averaging these data for each digitisation session, it was possible to build the "gold standard" as well as its statistical variation. Each dummy was photographed in two separate occasions and landmarks digitised twice for each capture. The 184 digitisations obtained with PhotoModeler ${ }^{\circledR}$ Scanner enabled to extract data about the operator error and the reproducibility error after coordinate superimposition performed using Orthogonal Procrustes Analysis [22]. Finally, the same best-fit procedure was employed again to align photogrammetric coordinates with the CMM "gold standard" in order to assess the system error.

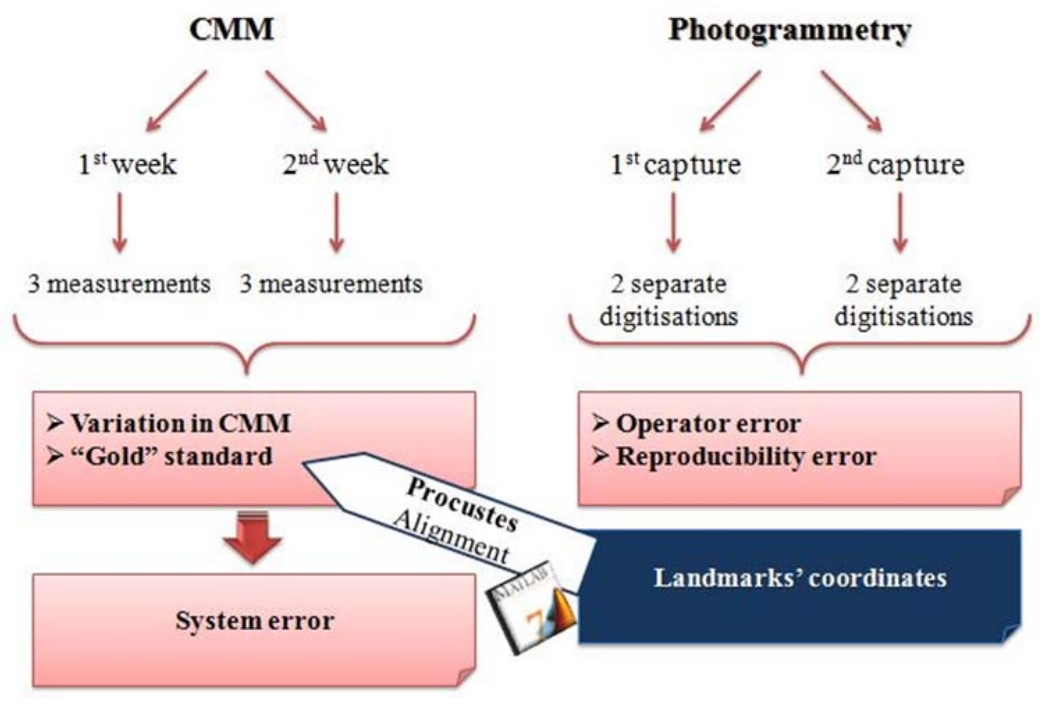

Fig. 2. General Method

At the earlier stage, the key step was marking specific anatomical facial landmarks. Based on Farkas' extensive work in the field [23], traditional anthropometry makes manual measurements using calipers, protractors or other instruments. However, modern contact and non-contact measuring methods enable increased level of precision through standardised assessment procedures. Hence, it is possible to collect a set of digitised landmarks from the soft-tissue face surface and use their spatial $X, Y, Z$ coordinates as reference points for calculating linear and angular distances [24]. Initial results were obtained using 23 facial landmarks marked as black-ink dots on each dummy. Different dot sizes where adopted for 'Mary' and 'Jack' dummies. More specifically, the anatomical landmarks used hereinafter were chosen among those proposed by de Menezes et al. (2009) [25] (Fig. 3, Table 1, ). 

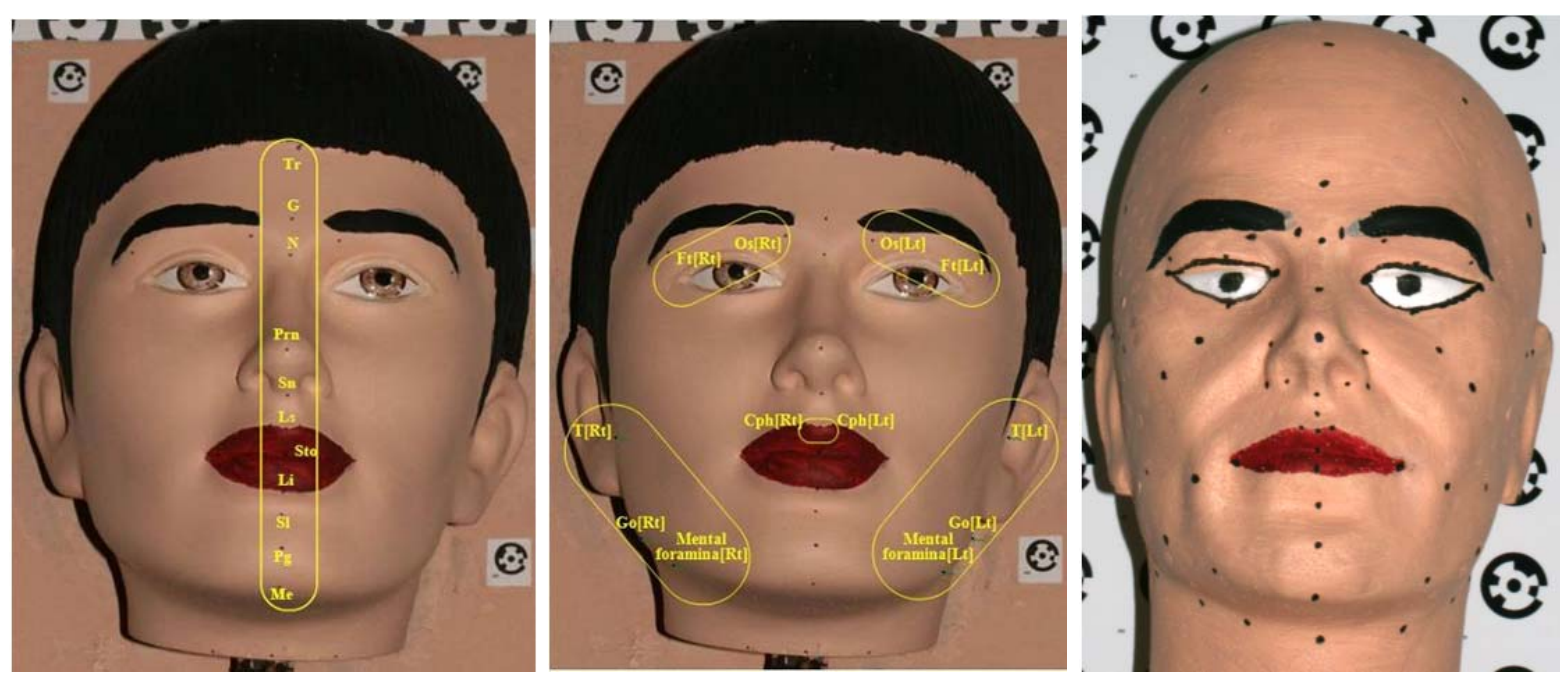

Fig. 3 .Facial landmarks on 'Mary' (left and centre) and 'Jack' (right) dummies.

Table 1. Midline landmarks description

\begin{tabular}{|c|c|c|}
\hline Name & Tag & Description \\
\hline Trichion & $\operatorname{Tr}$ & On the hairline in the middle of the forehead. \\
\hline Glabella & G & The most anterior midpoint on the fronto-orbital soft tissue contour. \\
\hline Nasion & $\mathbf{N}$ & $\begin{array}{l}\text { The midpoint on the soft tissue contour of the base of the nasal root at } \\
\text { the level of the fronto-nasal suture. }\end{array}$ \\
\hline Pronasale & Prn & The most protruded point of the apex nasi. \\
\hline Subnasale & Sn & $\begin{array}{l}\text { Midpoint on the nasolabial soft tissue contour between the columella } \\
\text { crest and the upper lip. }\end{array}$ \\
\hline Labiale superius & Ls & The midpoint of the upper vermilion line. \\
\hline Stomion & Sto & $\begin{array}{l}\text { The imaginary point at the crossing of the vertical facial midline and the } \\
\text { horizontal labial fissure between gently closed lips, with teeth shut in } \\
\text { natural position. }\end{array}$ \\
\hline Labiale inferius & Li & The midpoint of the lower vermilion line. \\
\hline Sublabiale & SI & $\begin{array}{l}\text { The most posterior midpoint on the labio-mental soft tissue contour that } \\
\text { defines the border between the lower lip and the chin. }\end{array}$ \\
\hline Pogonion & $\mathrm{Pg}$ & $\begin{array}{l}\text { The most anterior midpoint of the chin, located on the skin surface in } \\
\text { front of the identical bony landmark of the mandible. }\end{array}$ \\
\hline Menton & Me & Lowest median point on the lower border of the mandible. \\
\hline
\end{tabular}

Table 2. Paired landmarks description (left [Lt] and right side [Rt])

\begin{tabular}{lc|l}
\hline Name & \multicolumn{1}{c|}{ Tag } & \multicolumn{1}{c}{ Description } \\
\hline $\begin{array}{l}\text { Orbitale superius } \\
\text { Frontotemporale }\end{array}$ & $\begin{array}{c}\text { Os[Lt], Os[Rt] } \\
\text { Ft[Lt], Ft[Rt] }\end{array}$ & $\begin{array}{l}\text { Highest point on the lower border of the eyebrow. } \\
\text { On each side of the forehead, laterally from the } \\
\text { elevation of the linea temporalis. }\end{array}$ \\
$\begin{array}{l}\text { Christa philtri } \\
\text { Oph[Lt], Cph[Rt] } \\
\text { Tragion }\end{array}$ & $\begin{array}{l}\text { T[Lt], T[Rt] } \\
\text { the vermilion line. }\end{array}$ \\
$\begin{array}{l}\text { Gonion } \\
\text { Mental foramina }\end{array}$ & $\begin{array}{l}\text { Most latergal point on the mandibular angle. } \\
\text { Located on the skin surface in front of the identical } \\
\text { bony landmark of the mandible. }\end{array}$ \\
\hline
\end{tabular}

Accuracy indicators were computed using landmarks from both dummies at the same time. Thus, calculations produced absolute error evaluations. Subsequently, the same calculation protocol was 
used to produce separate indicators for each dummy. The aim was to appraise which of the two gave best results in terms of accuracy. This investigation appeared to be particularly sensible since the intrinsic differences between the two could be considered as a potential source for dissimilar results. The first part of the photogrammetric digitisation consisted in taking different photographs of the two dummies. They were marked, fixed to the tripod and adequately positioned relatively to the cameras. After determining the right lighting condition, the main shooting parameters were found by a trial and error approach, which led to an aperture value Av equal to f/11, a shutter speed Tv of $1 / 100$ s and a ISO rating of 100 . Each camera was manually focused on a specific target: UL and LL on the right cheek, UR and LR on the left cheek, CN either on the mouth or on the tip of the nose. Finally, after cameras' synchronisation, five simultaneous photos could be taken.

Photogrammetric digitisations were performed using PhotoModeler® Scanner Sub-pixel Mode target marking. Therefore, each facial landmark was manually selected and then detected by the software centroid search algorithm. The 3D coordinates were stored in a database for later analysis. PhotoModeler® Scanner can automatically assess precision only, since accuracy has to be established employing external check-distances. The software provides precision values based on the post-processing concentration matrix of the 3D object points. PhotoModeler ${ }^{\circledR}$ Scanner outputs precision values at $1-\sigma$ for each axis, or the $68 \%$ probability threshold. However, for each 3D point overall precision measures had to be taken into account, namely the precision vector length, the maximum and RMS residual and the tightness of the light rays.

A DeMeet- 400 by Schut Geometrische Meettechniek bv (accuracy $0.5 \mu \mathrm{m}$ ) was employed for CMM digitisations. The averaged set of CMM measurements was used as a "gold standard", against which the accuracy of the photogrammetric system would be assessed.

\section{Results}

\subsection{Precision}

3D face modelling entails PhotoModeler ${ }^{\circledR}$ Scanner processing algorithm should solve 3D points. Consequently, their precision is estimated in all three dimensions. Precision values are displayed as ellipsoids, which are iso-surfaces of constant probability error. In other words, every point on one ellipsoid represents a constant 1- $\sigma$ probability of the point position. Therefore, if an ellipsoid resembles a sphere, then the actual point position is equally expected in all three directions. On the contrary, if it has a prominent oval shape, then the point is less precise in one direction. Besides, the relative size of the ellipsoids gives a quick visual prompt about which points are more precise or imprecise: the larger the ellipsoid, the greater the probability that point will be imprecise. However, quantitative figures are found in point tables. The overall precision value, however, is expressed through the Precision Vector Length as:

$$
\text { Precision vector length }=\left[(\mathrm{X} \text {-precision })^{2}+(\mathrm{Y} \text {-precision })^{2}+(\mathrm{Z} \text {-precision })^{2}\right]^{1 / 2}
$$

Besides, the residual error is computed through its mean (RMS) and maximum values. Because of inaccuracies in camera station orientation and point marking, the light rays will not intersect perfectly. Thus, the ray tightness number indicates how well they intersect. The smaller the number, the better the intersection is. Typically, in high-accuracy projects, the tightness value should be about $0.01 \%$, meaning the gap between light rays is one-thousandths of the largest project extent.

The best angle between the photographs that image a specified point is also included. In fact, when the light rays intersect at a small angle, the position of the computed point tends to be less accurate since it is more prone even to small errors. Let $\alpha_{\mathrm{ijk}}$ be the angle between light rays $\mathrm{j}$ and $\mathrm{k}$ intersecting in point $\mathrm{i}$. Then, the best angle is equal to the maximum value resulting from the relation:

$$
\forall \mathrm{i} \text { : maximum angle }=\max \left\{\alpha_{\mathrm{ijk}}\right\}_{\mathrm{j}, \mathrm{k}}
$$

Where " $\mathrm{k}$ " is the dummies, " $\mathrm{j}$ " is the digitalisation number and " $\mathrm{i}$ " is the landmark number.

\subsection{Operator error}

The operator error is the amount of error associated with repeatedly digitising landmarks on the dummies' faces using PhotoModeler ${ }^{\circledR}$ Scanner. This type of error depends on target sub-pixel marking. In fact, point marking requires the user to zoom in and click in order to mark the point location. However, a human operator is bound to bear a certain amount of inaccuracy in this operation. To quantify the operator error, the Euclidean distance $\left(d_{i, j}\right)_{k}$ between repeatedly digitised 
sets of landmarks $\left(P_{i, j}\right)_{k}$ and $\left(P_{i, j}^{\prime}\right)_{k}$ was calculated. In the end, the operator error measure was the mean value $d_{i, j}$ taken over the 2 dummies

From mean values $\left(\mathrm{d}_{i, 1}\right)_{\mathrm{k}}$ and $\left(\mathrm{d}_{\mathrm{i}, 2}\right)_{\mathrm{k}}$ it was possible to calculate the following statistical data referring to the operator error over the two captures and for 23 facial landmarks.

In order to understand whether 'Mary' or 'Jack' gave best results in term of operator error, the aforementioned calculations were repeated separately for each dummy. The only difference consisted in averaging over the number of digitisations $\mathrm{j}$.

A mean value of $0.059 \mathrm{~mm}$ for the operator error was found. More specifically, this error is distributed between the dummies, namely $0.061 \mathrm{~mm}$ for 'Jack' and $0.052 \mathrm{~mm}$ for 'Mary'. Moreover, some landmarks (Sto, $\mathrm{Li}, \mathrm{T}[\mathrm{Lt}]$ and $\mathrm{T}[\mathrm{Rt}]$ ) present a greater departure from the mean value due to their position on the dummies' face surface.

\subsection{Reproducibility error}

The system robustness with regard to landmarks identification could be evaluated assessing the reproducibility error. It is the magnitude of error associated with re-capturing and re-digitising the dummies at two different occasions. More precisely, this study assessed the repeatability error, or test-retest reliability. In fact, according to ISO/IEC Guide 99:2007 [26], the authors considered the variation in measurements taken by a single operator and instrument on the same item and under the same conditions. The two initial sets of coordinates $\left(P_{i, j}\right)_{k}$ and $\left(P_{i, j}\right)_{k}$ from each $j^{- \text {th }}$ photo capture session were averaged. This produced two 3D configurations where $\left(\operatorname{Pm}_{\mathrm{i}, \mathrm{j}}\right)_{1}$ and $\left(\mathrm{Pm}_{\mathrm{i}, \mathrm{j}}\right)_{2}$ were the midpoints obtained as

$$
\left(\mathrm{Pm}_{\mathrm{i}, \mathrm{j}}\right)_{\mathrm{k}}=1 / 2\left[\left(\mathrm{P}_{\mathrm{i}, \mathrm{j}}\right)_{\mathrm{k}}+\left(\mathrm{P}_{\mathrm{i}, \mathrm{j}}^{\prime}\right)_{\mathrm{k}}\right]
$$

Between one capture and the other, the dummies had been moved away from their scanning position and then replaced. Therefore, inevitable changes in terms of orientation occurred. Consequently, the $3 \mathrm{D}$ configurations had to be brought back into a common reference system. This registration process was solved by means of Orthogonal Procrustes Analysis (OPA) and performed by Matlab $₫$ version 7.1. The Euclidean distance $\left(\mathrm{d}_{\mathrm{i}}\right)_{\mathrm{k}}$ between the two sets of aligned coordinates was calculated. Finally, the repeatability measure was expressed as the mean distance $d_{i}$ over the $k$ index, having a mean value of $0.106 \mathrm{~mm}$ and $0.074 \mathrm{~mm}$ for 'Mary' and 'Jack' respectively. Similarly to previous considerations on the operator error, the reproducibility error increases in value for those points located in a prominently lateral position (Sto, $L i, T[L t], T[R t]$ ).

\subsection{Variation in CMM}

The amount of error in CMM measurements between different digitisations of the same point was expressed through statistical variation. Points had been digitized twice three times each. Averaging these measurements each time produced two sets of "gold standards" per dummy. After averaging over the $\mathrm{k}$ index, OPA alignment was performed. Finally, the CMM variation was computed as the Euclidean distance between each pair of measurements. Similarly, additional results were achieved separately for each dummy.

\subsection{System error}

Global information about the photogrammetric system accuracy in facial landmark digitization derived from the comparison with the CMM measurements. These were averaged over each dummy and the mean coordinates $\left(\mathrm{P}_{\mathrm{i}}\right)_{\mathrm{k}}$ were assumed as the "gold standard". OPA was then employed to align the CMM "gold standard" with the photogrammetric digitalisations. After OPA, the Euclidean distance $\left(\mathrm{d}_{\mathrm{i}, j}\right)_{\mathrm{k}}$ between the matched coordinates could be calculated. First, the distances were averaged over the two digitisations producing $\left(\mathrm{d}_{\mathrm{i}, \mathrm{j}}\right)_{\mathrm{k}}$. Finally, the system error $\Delta_{\mathrm{i}, \mathrm{k}}$ was calculated averaging $\left(\mathrm{d}_{\mathrm{i}, \mathrm{j}}\right)_{\mathrm{k}}$ over the two dummies (Table 3 ). Similarly, additional results were achieved separately for each dummy (Table 4).

Table 3. Mean system error of two different captures $(\mathrm{mm})$

\begin{tabular}{c|c|c|}
\multicolumn{1}{c}{} & \multicolumn{2}{c}{ STATISTICS } \\
\cline { 2 - 3 } Mean values & 1st CAPTURE & 2nd CAPTURE \\
\cline { 2 - 3 } Max values & 0.424 & 0.426 \\
\cline { 2 - 3 } Min values & 0.778 & 0.711 \\
\cline { 2 - 3 } Standard deviations & 0.195 & 0.233 \\
\cline { 2 - 3 } & 0.151 & 0.135 \\
\cline { 2 - 3 }
\end{tabular}

Table 4. Mean system error for each dummy ( $\mathrm{mm})$

\begin{tabular}{c|c|c|}
\multicolumn{1}{c|}{} & \multicolumn{2}{c}{ STATISTICS } \\
\cline { 2 - 3 } & MARY & JACK \\
\cline { 2 - 3 } Mean values & 0.313 & 0.538 \\
\cline { 2 - 3 } Max values & 0.748 & 1.082 \\
\cline { 2 - 3 } Min values & 0.075 & 0.144 \\
\cline { 2 - 3 } Standard deviations & 0.150 & 0.258 \\
\cline { 2 - 3 } & &
\end{tabular}


The analysis of the points in Fig. 4 leads to the conclusion that the system error does not substantially change when it is computed at various times. Its mean value amounts to $0.425 \mathrm{~mm}$. The comparison between the dummies clearly shows the points of greater positive departure from average values, namely Sn and Os[Lt] for 'Mary', T[Lt] and Mental foramina[Lt] for 'Jack'.

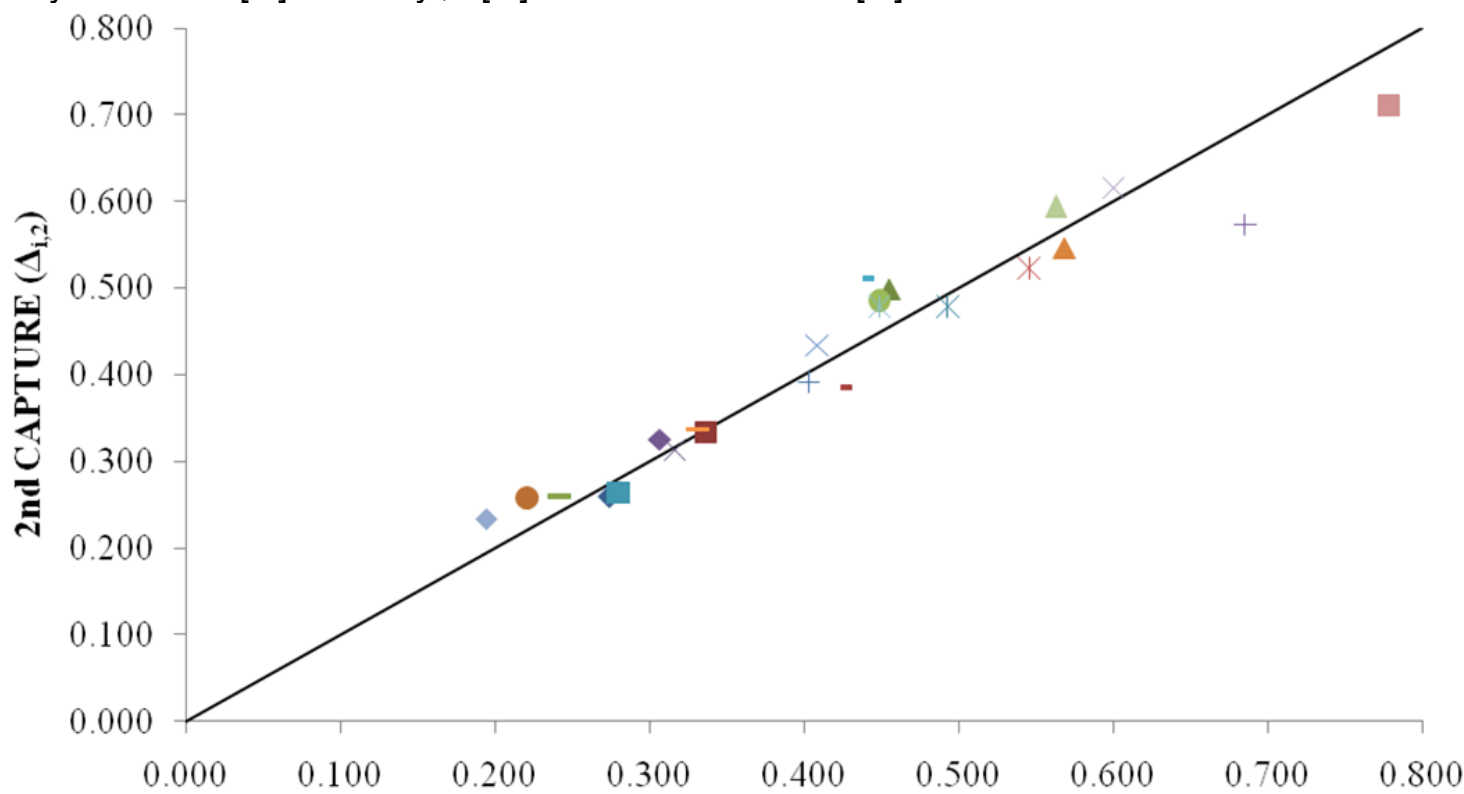

1st CAPTURE $\left(\Delta_{\mathrm{i}, 1}\right)$

$\begin{array}{llll}\bullet \mathrm{N} & \square \mathrm{Tr} & \Delta \mathrm{G} & \times \mathrm{Prn} \\ * \mathrm{Sn} & \bullet \mathrm{Ls} & +\mathrm{Sto} & -\mathrm{Li} \\ -\mathrm{Sl} & \diamond \mathrm{Pg} & \square \mathrm{Me} & \Delta \mathrm{Os}[\mathrm{Lt}] \\ \times \mathrm{Os}[\mathrm{Rt}] & * \mathrm{Ft}[\mathrm{Lt}] & \oplus \mathrm{Ft}[\mathrm{Rt}] & +\mathrm{T}[\mathrm{Lt}] \\ -\mathrm{T}[\mathrm{Rt}] & -\mathrm{Cph}[\mathrm{Lt}] & \diamond \mathrm{Cph}[\mathrm{Rt}] & \square \text { Mental foramina[Lt] } \\ \Delta \mathrm{Mental} \text { foramina[Rt] } & \times \mathrm{Go}[\mathrm{Lt}] & \times \mathrm{Go}[\mathrm{Rt}] & \end{array}$

Fig. 4 System error $(\mathrm{mm})$

\section{Discussion}

The experiments conducted in this study demonstrated that the implementation of PhotoModeler $®$ Scanner produced very precise landmark digitisations. The mean precision level achieved was $0.126 \mathrm{~mm}$ with a standard deviation of $0.013 \mathrm{~mm}$. Both confidence regions and point tables reveal that the less precise digitised landmark is the right gonion (Go[Rt]) for 'Mary', the right tragion (T[Rt]) for 'Jack'. The most plausible reason is that PhotoModeler's sub-pixel marking algorithm found it hard to recognize these points because of their lateral position. As a consequence, they were marked only on two photos each, resulting in too low angles $\alpha_{i j k}$. (Table 5) account for the best and worst results achieved in terms of precision vector length.

Table 5. Precision of vector length.

\begin{tabular}{c|ccll}
\hline \multicolumn{2}{c}{} & \multicolumn{1}{c}{ Value } & Point & Capture - Digitisation \\
\hline \multirow{2}{*}{ Best } & 'Mary' & $0.061 \mathrm{~mm}$ & Me & $2^{\text {nd }}$ capture $-1^{\text {st }}$ digitisation \\
value & 'Jack' & $0.104 \mathrm{~mm}$ & Mental foramina $[\mathrm{Lt}]$ & $1^{\text {st }}$ capture $-1^{\text {st }}$ digitisation \\
$\begin{array}{c}\text { Worse } \\
\text { value }\end{array}$ & 'Mary' & $0.350 \mathrm{~mm}$ & $\mathrm{Go}[\mathrm{Rt}]$ & $1^{\text {st }}$ capture $-2^{\text {nd }}$ digitisation \\
\hline
\end{tabular}


As for accuracy, the face scanning system used has proved to yield better results compared to those discussed in similar studies [11, 15]. In fact, the scientific approach herein discussed has led to a negligible operator error within $0.059 \mathrm{~mm}$.

As for reproducibility, a measurement may be said to be repeatable when the repeatability coefficient is smaller than some agreed limit. The British Standards Institution [27] assumes as repeatability coefficient the measure which represents the value below which the absolute difference between two repeated test results may be expected to lie with a probability of $95 \%$. In other words, we expect that $P\left(\left|d_{i}-\mu\right|<2 \sigma\right)$. The results achieved in this study, proved to be consistent with this limitation (Fig. 5). In fact, 22 landmarks out of 23 , or equally $96 \%$ of points, lie within the $2 \sigma$ interval. Only the stomion, with a $d_{i}=0.197 \mathrm{~mm}$, departs from the upper $2 \sigma$ limit $(0.191 \mathrm{~mm})$ of the negligible $3 \%$. Comparing the dummies, 'Jack' outperformed 'Mary' of about $43 \%$ in terms of mean value.

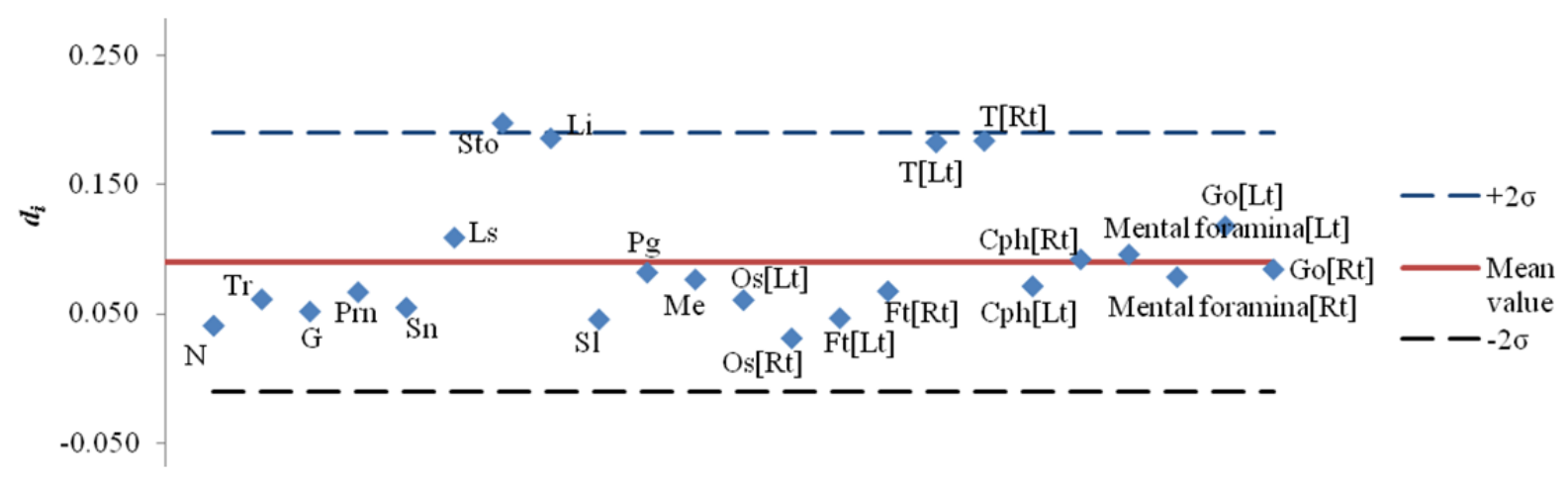

Fig. 5. Reproducibility error $(\mathrm{mm})$

As discussed before, the authors found that only some landmarks (Sto, Li, T[Lt], T[Rt]) show a greater departure from the mean value. A reasonable explanation may lie in the point acquisition method at software level. In fact, PhotoModeler ${ }^{\circledR}$ Scanner sub-pixel point marking uses a centroid search algorithm. It computes the centre of gravity of a disk-like 2D object through a floating-point averaging of all points in the disk as recognized by binary thresholding of the image. From a practical point of view, the user zooms in where the landmark is positioned and marks it with a circle. The algorithm best recognizes circular shapes if there is enough contrast. In photos, both points $T[L t]$ and $T[R t]$ appear as deformed because of their lateral position on the face surface. Thus, the sub-pixel marking algorithm identifies them as ellipses rather than circles, which results in the loss of precision. On the other hand, Sto and Li are characterised by low contrast being located on the lips. In conclusion, the approximation introduced by the software marking method seems to affect both operator and reproducibility error.

All data were further analysed in order to establish whether the propagation of system error followed a normal distribution, as the majority of observational errors do, rather than any other particular statistical distribution. For this purpose, a normal probability plot was derived by plotting standardized $z_{i}$ values against $\Delta_{i}$ : data lie quite close to the straight line representing the theoretical normal distribution. Therefore, it could be concluded that data are adequately consistent with a sample from a normal distribution. For the sake of completeness, some important model-fitting indexes were calculated. Most importantly, the square of the correlation coefficient $R^{2}(\approx 0.97)$ predicted fairly good approximation between normal distributed and real data. Compared to the mesokurtic normal distribution (i.e. null kurtosis), observed data do have a slight negative excess kurtosis $(-0.50)$, which suggested a lower and wider peak around the mean. A $\sim 0.38$ skewness value qualitatively indicated that the tail on the right side was slightly longer than the left side and the bulk of the values lay to the left of the mean. In other words, there exists a small symmetry departure from a normal distribution. Finally, it could be concluded that the system error was produced by a large number of small effects acting additively and independently, and thus its distribution could be close to normal. This conclusion, incidentally, is mathematically consistent with the central limit theorem. Assuming $\Delta_{\mathrm{i}}$ as a random normally distributed variable, the statistics shown in Table 6 could be drawn.

Potential follow-up studies will investigate further the reasons why 'Mary' outperformed 'Jack' of about $72 \%$ in terms of mean system error. 
[5] Galantucci L. M, Percoco G., Di Gioia E., (2010): "Low cost 3D face scanning based on landmarks and photogrammetry: A new tool for a surface diagnosis in orthodontics", Intelligent Automation and Computer Engineering, Lecture Notes in Electrical Engineering, Intelligent Automation and Computer Engineering Series, Ao, Sio-long; Castillo, Oscar; Huang, Xu (Eds.), Springer, ISBN: 978-90-481-3516-5, , Volume 52, cap. 8, pp. 93106, DOI: 10.1007/978-90-481-3517-2_8

[6] Ma L., Xu T., Lin J., (June 2009), "Validation of a three-dimensional facial scanning system based on structured light techniques", Computer Methods and Programs in Biomedicine, Volume 94, Issue 3, pp.290-298.

[7] Enciso R., Shaw A., Neumann U., Maha J., (2003), "3D head anthropometric analysis" In proceedings of the international society for optical engineering, SPIE Medical Imaging, San Diego,California, Issue 5029, pp.590597.

[8] Derya Germec-Cakan, Canter Halil, Nur Burcu, Arun Tulin, (September 2010), "Comparison of Facial Soft Tissue Measurements on Three-Dimensional Images and Models Obtained With Different Methods", Journal of Craniofacial Surgery, Volume 21, Issue 5, pp 1393-1399.

[9] Lee JY, Han Q, Trotman CA., (2004 Oct), "Three-dimensional facial imaging: accuracy and considerations for clinical applications in orthodontics". Angle Orthod.;74(5):587-93.

[10] Ayoub A., Garrahy A., Hood C., White J., Bock M., Siebert J.P., Spencer R., Ray A., (September 2003), "Validation of a Vision-Based, Three-Dimensional Facial Imaging System." Cleft Palate-Craniofacial Journal, Vol. 40 No. 5.

[11] Ayoub A. F., Xiao Y., Khambay B., Siebert J. P., Hadley D., (April 2007), "Towards building a photo-realistic virtual human face for craniomaxillofacial diagnosis and treatment planning", Int. J. Oral Maxillofac. Surg. 2007, Volume 36, pp.423-428,

[12] Boyadjiev SA., (March 2007), "Genetic analysis of non-syndromic Craniosynostosis" Orthod Craniofacial Res, Volume 10, pp.129-137.

[13] Hammond P., Hutton T.J., Allanson J.A., Shaw A., Patton M., "3D Digital Stereo Photogrammetric Analysis of Face Shape in Noonan Syndrome", From the proceedings of the British Human Genetics Conference 2002, York

[14] Rangel F.A., Maal T.J.J., Bergé S.J. et al., (December 2008), "Integration of digital dental casts in 3dimensional facial photographs", American Journal of Orthodontics \& Dentofacial Orthopedics, Volume 134, Issue 6 , pp.820-826.

[15] Khambay B., Nairn N., Bell A., Miller J., Bowman A. and Ayoub A.F., (January 2008), "Validation and reproducibility of a high-resolution three-dimensional facial imaging system." British Journal of Oral and Maxillofacial Surgery, Volume 46, Issue 1, Pages 27-32.

[16] Winder R.J., Darvann T.A., McKnight W., Magee J.D.M., Ramsay-Baggs P., (January 2008), "Technical validation of the Di3D stereophotogrammetry surface imaging system" British Journal of Oral and Maxillofacial Surgery, Volume 46, Issue 1, Pages 33-37.

[17] Lübbers H.T., Medinger L., Kruse A., Grätz K.W., Matthews F., (May 2010), "Precision and Accuracy of the 3dMD Photogrammetric System in Craniomaxillofacial Application", Journal of Craniofacial Surgery: - Volume 21 - Issue 3 - pp 763-767

[18] Lübbers H.T., Medinger L., Kruse A.L., Grätz K.W., Obwegeser J.A., Matthews F., (2011 Jan 12), "The influence of involuntary facial movements on craniofacial anthropometry: a survey using a three-dimensional photographic system.", Br J Oral Maxillofac Surg.

[19] Deli R., Di Gioia E., Galantucci L.M., Percoco G., (January 2010), "Automated landmarks extraction for orthodontic measurement of faces using the 3 cameras photogrammetry methodology", Journal of Craniofacial Surgery, Vol.21, Issue 1, pp.87-93.

[20] Deli R., Di Gioia E., Galantucci L.M., Percoco G., (January 2011), "Accurate facial morphology measurements using a 3-camera photogrammetric method", Journal of Craniofacial Surgery, Vol. 22, Number 1, 22 (1): 54-59.

[21] Galantucci L.M., Lavecchia F., Percoco G., (2010), "A simple photogrammetric system for automatic capture and measurement of facial soft tissues during movement", Innovative Development in Design and Manufacturing, Paulo Jorge Bártolo et al. (eds), Taylor \& Francis Group, London, ISBN 978-0-415-87307-9 (Hbk), 978-0-20385947-6 (Ebook), pp. 151-156.

[22] Schonemann P.H., (March 1966) "A generalized solution of the orthogonal Procrustes problem", Psychometrika, v.31, n.1, pp.1-10.

[23] Farkas L.G., (1994), "Anthropometry of the head and face, 2nd ed., Raven Press, New York,

[24] Sforza C., Ferrario V.F., (2006) "Soft-tissue facial anthropometry in three dimensions: from anatomical landmarks to digital morphology in research, clinics and forensic anthropology", J Anthropol Sci., v.84, pp.97-124. [25] de Menezes M., Rosati R., Allievi C., Sforza C., (2009), "A Photographic System for the Three-Dimensional Study of Facial Morphology", Angle Orthod., v.79, pp.1070-1077.

[26] ISO/IEC Guide 99-12:2007 International Vocabulary of Metrology - Basic and general concepts and associated terms (VIM).

[27] British Standards Institution Precision of test methods 1: Guide for the determination and reproducibility for a standard test method (BS 597, Part 1), London, BSI, 1975. 